\title{
Schuldenkrisen im Wandel: Das neue Zeitalter von Staatsbankrotten und Umschuldungen
}

\author{
Henrik Enderlein / Julian Schumacher / Christoph Trebesch
}

The changing world of sovereign debt crises: on a new age of sovereign defaults and debt restructuring

Abstract: This paper describes different forms of sovereign defaults and identifies changes since the mid-1990s. It shows why the topic is of relevance for international and comparative political economy but also points to possible reasons why the empirical literature is often inconclusive. Sovereign defaults stand for very different phenomena that are characterized here on the basis of eleven different criteria. The paper builds on three new datasets that are assessed descriptively and point towards quite significant changes over time. Economic characteristic of sovereign defaults, but also the legal and political-institutional context and changing conditions in international financial markets all indicate that there have been quite important changes that should be taken up by research in political economy.

Keywords: Debt crises, sovereign defaults, sovereign debt restructuring, financial crises, international political economy

Schlagwörter: Schuldenkrisen, Staatsbankrotte, Finanzmarktkrisen, internationale politische Ökonomie.

\section{Einleitung'}

Wenn ein souveräner Staat nicht mehr willens oder in der Lage ist, die finanziellen Forderungen seiner Gläubiger fristgerecht $\mathrm{zu}$ bedienen, entsteht die Situation eines Staatsbankrotts oder einer Umschuldung. In der wirtschaftspolitischen Praxis, aber auch in der Forschung der politischen Ökonomie, ist diese wahrscheinlich radikalste Form der „Lösung“ einer Schuldenkrise in den vergangenen Jahren wieder zu einem Kernthema geworden. Das liegt nicht nur daran, dass mit der „großen Rezession“ auch die Schuldentragfähigkeit vieler Länder in Frage gestellt wurde, sondern - wie dieser Aufsatz argumentiert - auch an veränderten institutionellen Rahmenbedingungen der Interaktion zwischen privaten Gläubigern und staatlichen Schuldnern.

Dieser Aufsatz stützt sich auf drei vollkommen neue Datensätze zu Staatsbankrotten und Schuldenkrisen und wertet diese auf Veränderungsprozesse und -dynamiken hin aus. Er verfolgt dabei erstens das Ziel, zu zeigen, dass unterschiedliche

1 Die Forschungsarbeiten an diesem Aufsatz wurden durch die DFG im Rahmen des Sonderforschungsbereichs 700 gefördert. Wir danken Alexander Agronovsky, Andreea Firca, Paul Jasper, Inga Karrer, Said Khalid Scharaf, Lilly Schoen, Lina Tolvaisaite und Alexander Vatagin für ihre exzellente Unterstützung an verschiedenen Stellen der Forschung. Teile der Forschung sind entstanden während der Aufenthalte von Henrik Enderlein als Pierre Keller Gastprofessor an der Harvard Universität und am Weatherhead Center of International Affairs, von Julian Schumacher an der Columbia Universität und von Christoph Trebesch beim Internationalen Währungsfonds. 
Staatsbankrotte völlig unterschiedliche Charakteristika aufweisen, in der einschlägigen Literatur deshalb nicht als vergleichbar behandelt werden sollten. Er stellt elf Kriterien zur politökonomischen Einordung von Staatsbankrotten und Umschuldungen auf, die dank der hier verwendeten Datensätze in die zukünftige empirische Arbeit einfließen können. Er zeigt zweitens, dass auch innerhalb dieser unterschiedlichen Kriterien in den vergangenen Jahrzehnten sehr wichtige Veränderungen festzustellen sind, die auf ein „,neues Zeitalter" von Staatsbankrotten hindeuten, das sich seit der Mitte der 1990er Jahre herausgebildet hat.

Die Veränderungen beziehen sich auf vier Aspekte. Erstens: veränderte Charakteristika der ökonomischen Rahmendaten der Staatsbankrotte selber, weil diese inzwischen zwar weniger häufig sind, die Schuldenschnitte („Haircuts“) auf Anleihen aber größer geworden sind und sich auch die Art der Umschuldung deutlich verändert hat (Fokus auf Nominalwertkürzungen anstatt auf Fristenverlängerungen). Zweitens: Veränderungen in der Struktur der internationalen Finanzmärkte, vor allem was die Art der Staatsschulden betrifft (mehr Anleihen, weniger direkte Bankkredite) oder auch die Ansteckungsgefahren (systemische Relevanz einzelner Staaten). Drittens steht das neue Zeitalter für einen veränderten Rechtsrahmen bei den Umschuldungen, weil sich in den vergangenen Jahrzehnten eine stille Revolution in der internationalen Rechtsprechung zu Umschuldungen ergeben hat, die eine Kernannahme der klassischen politischen Ökonomie, Staatsschulden entzögen sich dem Zugriff von Gerichten, zumindest infrage stellt. Anlegerforderungen können inzwischen einfacher eingeklagt werden als in der Vergangenheit, und jede zweite Umschuldung ist heute von einer Anlegerklage betroffen. Viertens, und hier von besonderer Relevanz für die politikwissenschaftliche Literatur: Veränderungen in den politisch-institutionellen Rahmenbedingungen des Umschuldungsprozesses, vor allem was die Verhandlungsmodalitäten und mit ihr die Dauer der Umschuldungen betrifft (klarer Trend zu schnelleren Umschuldungen), aber auch den Kooperationsgrad der Regierungen gegenüber den Gläubigern (ein Trend hin zu einer Polarisierung zwischen stark konfliktuell vs. stark kooperativ).

Insgesamt hat der Aufsatz einen Überblickscharakter und will zeigen, dass es sowohl im Längs- als auch im Querschnitt der Forschung zu Staatsbankrotten dringenden Nachholbedarf gibt, der durch die hier vorgestellten Datensätze begonnen werden kann.

Der Aufsatz beginnt mit einer kurzen Literaturübersicht zur politikwissenschaftlichen und polit-ökonomischen Literatur zu Staatsbankrotten (Abschnitt 2) und stellt dann eine Typologie der wichtigsten Charakteristika von Staatsbankrotten auf (Abschnitt 3), ehe dann die vier zentralen Veränderungsprozesse herausgearbeitet werden (Abschnitt 4). Die Schlussfolgerung untersucht Implikationen der Arbeit für die polit-ökonomische Forschung.

\section{Staatsbankrotte in der politischen Ökonomie}

Aus stark zugespitzter Perspektive heraus betrachtet steht jedes Land in einer finanziellen Notlage vor der Wahl zwischen einem nationalen Konsolidierungskurs, in der Regel verbunden mit hohen politischen Kosten durch steigende Steuern 
und/oder geminderte Ausgaben, und der Aufkündigung des Schuldendienstes an die Gläubiger.

Ein Staatsbankrott tritt ein, wenn eine existierende Staatsschuld in eine neue Staatsschuld zu schlechteren Konditionen für die Gläubiger umgewandelt wird. Die Art der Umwandlung (z. B. unilateral oder ausgehandelt) ist dabei bereits Teil der Analyse unterschiedlicher Typen von Staatsbankrotten, die weiter unten vertieft beschrieben wird.

Von besonderer Bedeutung bei Staatsbankrotten ist die außergewöhnliche Rechtslage bei Staatsschuldforderungen im Gegensatz zu Forderungen an private Unternehmen. Für letztere gibt es Insolvenzverfahren unter der Aufsicht nationaler Gerichte, die den Gläubigern in der Regel streng definierte Zugriffsrechte auf die Aktiva des Unternehmens einräumt. Bei Staatsschulden sind solche Zugriffe kaum möglich, weil sich Staatsbesitz vor allem auf dem eigenen Territorium befindet (oder dieses Territorium der Staatsbesitz ist - BILD forderte während des Staatsbankrotts Griechenlands den Verkauf griechischer Inseln ein...[BILD 2010]) und weil im Ausland befindliche Aktiva von Staaten in der Regel durch Immunität besonders geschützt sind (z. B. Botschaften). Zudem gibt es kein internationales Insolvenzgericht, das den Staaten übergeordnet wäre. Trotzdem sind Staatsanleihen eines der größten Anlageinstrumente der Weltfinanzmärkte.

Aus der Sicht politikwissenschaftlicher Forschung und der politischen Ökonomie sind drei zentrale Fragen bei der Analyse von Staatsbankrotten relevant.

1. Warum kann es Staatsschulden überhaupt geben, wenn die rechtliche Einforderung von Ansprüchen aufgrund der Staatssouveränität kaum möglich ist?

2. Welche politisch-institutionellen oder ökonomischen Faktoren können erklären, warum staatliche Schuldner sich entscheiden, Gläubigerforderung nicht mehr zu bedienen?

3. Welche politisch-institutionellen oder ökonomischen Faktoren können erklären, in welcher Form ein Staatsbankrott oder eine Umschuldung durchgeführt wird?

Auch wenn die Fragen sehr unterschiedlicher Natur sind, werden Sie in der Forschung oft gemeinsam beantwortet. Vor allem die ersten beiden Fragen stehen in engem Zusammenhang, da das Fehlen starker Rechtsmittel stets alternative politisch-institutionelle oder ökonomische Durchsetzungsfaktoren erfordert. Die dritte Frage ist hingegen ein Desiderat, das in diesem Aufsatz in den Vordergrund gerückt wird.

Doch zuvor zu den beiden ersten Fragen. Die Interaktion von Schuldnerregierung und Geldgebern ist von einem Zeitinkonsistenzproblem gekennzeichnet. Obwohl sowohl die Regierung als auch Investoren vom Zustandekommen einer Transaktion (Verschuldung) profitieren würden, kann die Regierung die Rückzahlung zu einem späteren Zeitpunkt nicht glaubwürdig garantieren. Daher müssen alternative Verpflichtungsmechanismen existieren. Die politökonomische Literatur beschreibt das Schuldner-Gläubiger-Verhältnis daher häufig als Reputationsspiel (Eaton u. Gersovitz 1981; Bulow u. Rogoff 1989; eine Übersicht geben Panizza et 
al. 2009; siehe auch Tomz 2007). ${ }^{2}$ Private Kreditgeber ziehen ihr Vertrauen in den Schuldendienst durch die Regierung aus der Annahme, die Schuldnerregierung wisse, dass eine Zahlungseinstellung ihren zukünftigen Zugang zu Kapitalmärkten entweder verteuern oder gänzlich unmöglich machen könnte. Die Logik der Interaktion stützt sich auf den Vergleich zwischen langfristigen Kosten und kurzfristigen Gewinnen: Weil ein Staatsbankrott die Risikoprämie und damit die Kosten zukünftiger Schuldenaufnahmen für das Land nachhaltig erhöht (siehe vor allem Cruces u. Trebesch 2013), zieht die Regierung eine Fortsetzung des Schuldendiensts in der Regel vor und wählt den Weg nationaler Konsolidierung. Dieser Ansatz kommt zu der Schlussfolgerung, dass ein gut funktionierendes Marktumfeld für Staatsanleihen die glaubwürdigste Garantie dafür bereitstellt, dass sich Schuldnerregierungen nicht für einen Zahlungsstopp oder ein Umschuldung entscheiden. Die „compliance“ resultiert daher aus Marktanreizen und bedarf in der Regel keiner zusätzlichen institutionellen oder regulativen Instrumente.

Dennoch kommt es recht häufig zu Staatsbankrotten. Die hier vorgestellten Datensätze zählen 187 Staatsbankrott-Fälle seit den 1970er Jahren, wobei viele dieser Fälle über mehrere Jahre andauern, die Anzahl der Jahres-Fälle also deutlich höher ist. Warum diese Häufigkeit?

Aus politökonomischer Sicht liegt es nahe, anzunehmen, dass nationale politische Variablen einen großen Anteil an den Erklärungen dafür haben, dass Gläubiger einzelnen Ländern mehr Vertrauen zukommen lassen und Regierungen sich immer wieder für die Einstellung des Zahlungsdiensts entscheiden. In der Tat ist davon auszugehen, dass der Zeithorizont im Zentrum der Regierungsüberlegungen steht, wenn ein Land mit Problemen der Staatsfinanzen und deshalb auch mit Problemen in der Aufrechterhaltung des Schuldendienstes konfrontiert ist. In einer Krise gerät die Regierung unter den Druck der Bevölkerung, nationalen wirtschaftspolitischen Bedürfnissen Vorrang zu geben, und nicht zum Zweck des externen Schuldendienstes die Steuern zu erhöhen oder Staatsausgaben massiv zu senken (Ponticelli u. Voth 2011). Dieser Druck konfrontiert die Regierung mit ihrem eigenen politischen Überleben (als Analogie kann hier auf die Literatur zu "audience costs" verwiesen werden, siehe z. B. Fearon 1994; Schultz 2001; Tomz 2004). Gleichzeitig weiß die Regierung, dass eine Zahlungseinstellung negative Konsequenzen für den zukünftigen Zugang zu externen Finanzierungsquellen nach sich zieht (die oben kurz beschriebenen Reputationskosten).

Die Regierung steht also vor einem Zielkonflikt, den sie unter der Berücksichtigung ihrer eigenen Handlungskapazität und des für sie entscheidenden Zeithorizonts zu lösen versucht. Dies wirft die Frage auf, welche Faktoren die Lösung dieses Zielkonflikts in die eine oder andere Richtung beeinflussen, also erklären können, warum manche Regierungen auf Probleme der Staatsfinanzen mit einer Zahlungseinstellung reagieren, andere aber einen nationalen Konsolidierungskurs bevorzugen.

2 Weitere Erklärungsmuster sind direkte Sanktionen (z. B. Handelsbarrieren, siehe Bulow u. Rogoff 1989 sowie Mendoza u. Yue 2012, oder „Kanonenbootdiplomatie“, siehe Mitchener u. Weidenmier 2005), oder negative Auswirkungen auf das einheimische Bankensystem (Gennaioli et al. 2014). 
Die politikwissenschaftliche Literatur hat sich vor allem im zurückliegenden Jahrzehnt deshalb immer intensiver damit auseinandergesetzt, welche nationalen institutionellen Rahmenbedingungen erklären könnten, warum Staaten sich für einen Staatsbankrott oder eine Umschuldung entscheiden. Dabei hat sie sich vor allem auf die Frage konzentriert, ob Demokratien, in denen Besitzstandsrechte von besonderer Bedeutung sind und Wahlen abgehalten werden, in denen aber auch mehr Veto-Spieler das Regierungshandeln beschränken (vgl. die detaillierte Beschreibung hierzu von Cordes 2014), mehr Gläubigervertrauen verdienen (der sog. „democratic advantage“, der auf eine historische Studie von North u. Weingast 1989 über England zurückgeht und von Schultz u. Weingast 2003 ausgearbeitet wurde). So überzeugend die Kausalmechanismen in dieser Literatur sein mögen, so wenig überzeugend sind die empirischen Ergebnisse (Archer et al. 2007; Biglaiser et al. 2012; Biglaiser u. Staats 2012). Bemerkenswert an der Literatur ist, dass sie einen starken Fokus auf die Debatten über die unabhängigen institutionellen Variablen legt (Definitionen und Typen von Demokratien, VetoSpieler [siehe hierzu Cordes 2014]), bei der abhängigen Variablen aber von übertriebener Vereinfachung geprägt ist.

In der Tat greifen weite Teile der empirischen Literatur auf eine binäre Unterscheidung von Staatsbankrott in „,ja“ oder "nein“ zurück (wie sie im Datensatz der Ratingagentur Standard and Poor's verfügbar ist). Auch die theoretische Literatur beschränkt ihre Perspektive oftmals auf diese Unterscheidung und impliziert damit in der Regel Verluste von 100 Prozent, wenn sie nicht - was selten der Fall ist - die Höhe des Schuldenschnitts oder andere Charakteristika als Schlüsselvariablen in die Untersuchung mit einbezieht. ${ }^{3}$

Gerade aus politikwissenschaftlicher und polit-ökonomischer Sicht ist diese Verengung aber nicht hilfreich. Denn selbst wenn für Marktteilnehmer die Inzidenz des Zahlungsausfalls an sich von Bedeutung ist und ggf. auch das Ausmaß, so können für politökonomische Analysen auch andere Charakteristika von Staatsbankrotten oder Umschuldungen in den Vordergrund rücken.

Vor diesem Hintergrund verfolgt dieser Aufsatz zwei Ziele. Er will erstens deutlich machen, dass die politökonomische Literatur über Staatsbankrotte oder Umschuldungen von übertriebener Vereinfachung geprägt ist, die erklären könnte, warum die empirischen Ergebnisse der Forschung oft widersprüchlich oder frei von klaren Schlussfolgerungen sind. Wer Staatsbankrotte erklären möchte, muss detaillierter klären, welcher Aspekt des Phänomens Staatsbankrott überhaupt untersucht werden soll. Der Aufsatz stellt deshalb Kriterien für eine politisch-institutionelle Typologie oder auch dringend notwendige empirische Klassifizierung von Staatsbankrotten vor. In einem zweiten Schritt weist der Aufsatz darauf hin, wie sehr sich Staatsbankrotte selbst innerhalb dieser Kriterien in den vergangenen Jahrzehnten verändert haben. Er zeigt damit, dass es sowohl im Quer- als auch im Längsschnitt dringenden Korrekturbedarf in der Forschung zu Staatsbankrotten gibt, die erst mit der Verfügbarkeit von Datensätzen wie den hier vorgestellten beginnen kann.

3 Ausnahmen sind Grossman u. Van Huyck 1985 oder Arellano et al. 2013. 


\section{Eine politisch-institutionelle Klassifizierung von Staatsbankrotten}

In diesem Abschnitt werden elf Schlüsselcharakteristika von Staatsbankrotten aufgeführt, die dazu dienen können, die Fälle nach bestimmten Kriterien zu untergliedern. Eine solche Typologie nach Kriterien, die ökonomische, finanzmarktbezogene, politisch-institutionelle und rechtliche Aspekte gemeinsam berücksichtigt, gibt es bislang in der Literatur nicht. Dabei ist gerade aus politisch-institutioneller Perspektive eine Gesamtschau dieser Teilaspekte wichtig. Die Klassifizierung stellt Einzelcharakteristika dar, die zukünftige Forschungsprojekte anleiten können. Diese Charakteristika sind als binäre Unterscheidungen aufgeführt oder als kontinuierliche Variablen. Wichtig ist, dass diese Charakteristika keine exklusiven Kategorien bilden, sondern sich überlagern können.

Dieser Aufsatz wertet bei der Einordung der Staatsbankrotte drei neue eigene Datensätze zu Staatsbankrotten oder Umschuldungen erstmals gemeinsam auf ihre politikwissenschaftlichen und polit-ökonomischen Implikationen hin deskriptiv aus. Diese Datensätze erfassen sowohl die institutionellen Charakteristika und Rahmenbedingungen der Umschuldungen und Staatsbankrotte (Enderlein et al. 2012), die wichtigsten ökonomischen Variablen (Cruces u. Trebesch 2013) als auch die rechtlichen Aspekte (Schumacher et al. 2014).

Die Datenbasis der drei Datensätze umfasst den Zeitraum der 1970er Jahre bis heute (mit leichter Varianz zwischen den Datensätzen, siehe die Detailbeschreibungen im Annex). Die Gesamtzahl der Staatsbankrotte in diesem Zeitraum beträgt 187 (Cruces u. Trebesch 2013) und verteilt sich auf ca. 72 unterschiedliche Staaten (wobei durch Regimewechsel oder territoriale Neuordnungen teilweise Unklarheiten darüber bestehen, ab welchem Punkt Staaten als unterschiedlich gelten können). Die von uns verwendeten Daten beziehen sich in der Regel nur auf Staatsbankrotte gegenüber externen Gläubigern. Auch bilaterale Umschuldungsabkommen zwischen Regierungen (vor allem im Rahmen des Pariser Clubs) beziehen wir nicht ein. ${ }^{4}$ Der Großteil der Staatsbankrotte fand in Entwicklungsund Schwellenländern statt. Nach IWF-Klassifizierung hatte es seit 1950 keinen Staatsbankrott in einer hoch entwickelten Volkswirtschaft gegeben (Das et al. 2012, S. 6), bis Griechenland 2012 die bis dato größte Umschuldung der Geschichte durchführte (Zettelmeyer et al. 2013).

Noch vor der Klassifizierung sind kurze grundsätzliche Anmerkungen zum Ablauf eines Staatsbankrotts notwendig. Wichtig ist zunächst die Unterscheidung zwischen dem Staatsbankrott an sich („default“) und der Umschuldung oder auch Restrukturierung der Staatsschulden („,restructuring“). Obwohl diese Begriffe oft sehr breit und als Synonyme verwendet werden, hat sich in der Fachliteratur die folgende Unterscheidung herausgebildet. Der Begriff Staatsbankrott beschreibt den Moment der Zahlungsunterbrechung oder die Ankündigung einer Umschuldung, wohingegen die Umschuldung selbst dann in einem zweiten Schritt erfolgt, wenn der Souverän den Gläubigern ein Angebot vorgelegt hat, dieses dann verhandelt wird, ehe es zu einer Annahme oder Ablehnung kommt und die Umschuldungsphase damit vorerst beendet ist (Das et al. 2012, S. 13). Gerade die

4 Es gab 447 Umschuldungsabkommen dieser Art seit 1950 (Das et al. 2012, S. 5). 
politikwissenschaftliche und politökonomische Literatur zu Staatsbankrotten wird einen Schwerpunkt auf diese Verhandlungsphasen legen (Enderlein et al. 2012). In einem weiteren Schritt erfolgt dann eine mögliche Rechtstreitphase, in der Gläubiger versuchen, ihre Ansprüche vor Gerichten geltend zu machen. Wie weiter unten beschrieben wird, ist diese Phase des Rechtstreits vor allem ein Charakteristikum neuerer Schuldenkrisen.

\section{Abbildung 1: Ablauf einer Umschuldung}
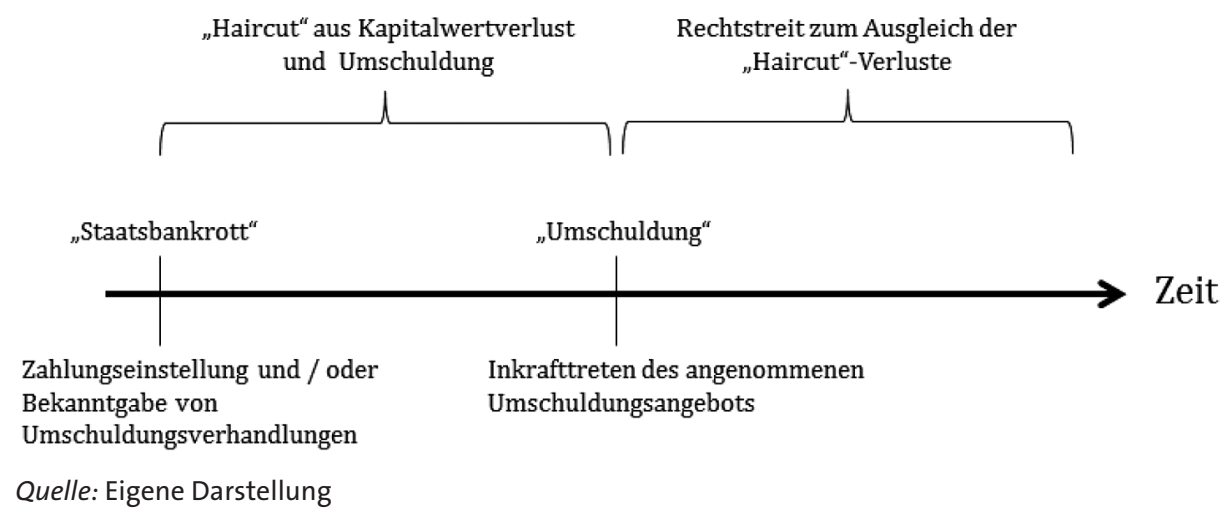

Die folgende Klassifizierung ist geordnet nach ökonomischen Kriterien, Finanzmarktkriterien, politisch-institutionellen Kriterien und rechtlichen Kriterien.

\section{1 Ökonomische Kriterien}

\subsubsection{Fristenverlängerung vs. Schuldenschnitt}

Entscheidet sich ein Souverän, die Forderungen der Gläubiger nicht mehr zu bedienen, dann gibt es grundsätzlich zwei Elemente: Erstens die Fristenverlängerung, bei der die Zahlungsflüsse in die Zukunft gestreckt werden, sodass für den Staat Einsparungen entstehen und für den Gläubiger Verluste (Barwertverluste). Zweitens den direkten Schuldenschnitt, bei der Zins- oder Nominalwertzahlungen direkt gekürzt werden. Aus Sicht des Gläubigers mit einem Fokus auf die Kapitalwertperspektive mag diese Unterscheidung nur begrenzt relevant sein Aus Sicht der Staaten ist die Perspektive eine andere, denn die Fristenverlängerung lässt den nominellen Schuldenstand unverändert, wohingegen der Schuldenschnitt diesen senkt. Die gängige Praxis, den offiziellen Schuldenstand in Nominalwerten anzugeben, ist jedoch nicht ohne Probleme. Sie kann dazu führen, dass die tatsächliche Tragfähigkeit der Verschuldung verschleiert wird (Dias et al. 2014). Der IWF hat sich daher kürzlich dafür ausgesprochen, schon verstärkt und zu früheren Zeitpunkten auf Laufzeitverlängerungen in Liquiditätskrisen zu drängen. Kapitalschnitte sollen hingegen weiterhin nur dann zum Einsatz kommen, wenn die Schuldendynamik eines Landes eindeutig untragbar geworden ist (IMF 2013, 
2014). Seit 1970 gab es 124 Fälle von Fristenverlängerungen und 63 Fälle von „echten“ Schuldenschnitten (Cruces u. Trebesch 2013).

\subsubsection{Kleiner vs. großer Schuldenschnitt}

Direkt mit dem Grundtypus des Staatsbankrotts verbunden ist die Frage nach der Höhe des Verlusts für die Anleihehalter und damit auch nach der Entlastung für den Staat. Die ökonomische Literatur kennt unterschiedliche Methoden zur Ermittlung des Verlusts, wobei gerade bei Fristenverlängerungen die Art der Berechnung des Kapitalwerts von erheblicher Bedeutung sein kann. Denn bietet eine Regierung ihren Gläubigern ein neues Papier mit einer neuen Laufzeit an, dann sind nicht nur die Opportunitätskosten unter Berücksichtigung des Risikos die zentrale Variable bei den Verlusten, sondern auch die Frage, ob der Vergleichswert der Nominalwert des alten Papiers ist oder der abgezinste Nominalwert des alten Papiers, also der Kapitalwert (Sturzenegger u. Zettelmeyer 2006). Letztere Methode hat sich durchgesetzt. Auf sie stützen sich auch die von uns verwendeten Daten. Seit 1970 beträgt der Durchschnittsverlust bei Staatsbankrotten 37 Prozent (Cruces u. Trebesch 2013). Die Varianz erstreckt sich über das komplette Spektrum von 0 Prozent (oder sogar Barwert-Gewinnen aus der Umschuldung) bis hin zu fast 100 Prozent Verlusten im Kontext von Entschuldungsinitiativen für Entwicklungsländer(Cruces u. Trebesch 2013).

\subsubsection{Kleine vs. große Umschuldungen (bzw. systemisch irrelevante vs. systemisch relevante Umschuldungen)}

Das Volumen der Umschuldungen ist für die Forschung von großer Bedeutung, denn eine kleine Umschuldung hat in der Regel weder für den internationalen Finanzmarkt noch für die Analyse der politischen Ökonomie von Schuldenkrisen die gleiche Bedeutung wie eine sehr große Umschuldung. Gerade bei einer immer engeren Verflechtung von Kapitalmärkten sind große Umschuldungen für den Weltanleihemarkt von größerer systemischer Bedeutung als kleinere Umschuldungen. Das Durchschnittsvolumen seit 1970 beträgt 6 Milliarden US Dollar (reale US Dollar von 1980), mit einer Varianz von etwa 10 Millionen US Dollar (Sao Tome \& Principe 1994 oder Honduras 2001) bis hin zur bislang größten Umschuldung der neueren Zeit in Griechenland 2012 mit einem Volumen von 260 Mrd. US Dollar (Cruces u. Trebesch 2013). Auch umgerechnet auf den Anteil des Schuldenschnitts am Bruttoinlandsprodukt ergeben sich starke Varianzen (Dias et al. 2012).

\subsection{Finanzmarktkriterien}

\subsubsection{Staatsbankrott auf Bankkredite vs. Staatsbankrott auf Anleihen}

Über einen langen Zeitraum wurden Staatsschulden vor allem als direkte Kredite von Banken oder Bankenkonsortien an Staaten ausgereicht. Diese Kredite standen zwar in den Bilanzen der Kreditinstitute, hatten aber keinen direkten Marktpreis, weil sie nur in seltenen Fällen gehandelt wurden. Mit der Erweiterung des 
globalen Anleihemarktes gingen immer mehr Staaten dazu über, ihre Schulden direkt am Kapitalmarkt aufzunehmen (siehe z. B. Tanaka 2006; Hale 2007). Die Unterscheidung ist im Kontext der prozeduralen Abläufe einer Umschuldung von erheblicher Bedeutung. Während ein Bankrott auf Bankkredite in der Regel im sog. „London-Club-Verfahren“ verhandelt wird, also die Gläubigerbanken eine Steuerungsgruppe mandatieren („Creditor Committee“), die die Umschuldungsmodalitäten aushandelt, wären Verhandlungen mit zahllosen Anleihehaltern in der Regel deutlich komplexer, was aber auch dazu führen kann, dass die Regierung den Umschuldungsprozess ohne direkten Verhandlungspartner sehr schnell und direkt durchführt (vgl. hierzu die Ausführungen bei Panizza et al. 2009, S. 672-674). Die Zahl der Anleiheumschuldungen ist deutlich geringer als die Zahl der Bankkreditumschuldungen (22 vs. 165 [Cruces u. Trebesch 2013]).

\subsubsection{Konzentrierte vs. diffuse Gläubigerstruktur}

Die Schuldenart (Bankkredit vs. Anleihe) ist allerdings nicht komplett gleichzusetzen mit der Struktur der Gläubiger. In den frühen 1980er Jahren wurden die Bankkredite von sehr kleinen, fast exklusiven Konsortien aufgelegt, die einen direkten Dialog mit den Schuldnerregierungen pflegten und dementsprechend eine Netzwerkbeziehung institutionalisierten, die im Fall der dann folgenden - vor allem lateinamerikanischen - Schuldenkrisen den Kooperationsgrad zwischen Schuldnern und Gläubigern maßgeblich prägte. In der wirtschaftspolitischen Praxis und polit-ökonomischen Forschung wurde die Gläubigerstruktur lange als Schlüsselvariable in Umschuldungsprozessen gesehen. Hintergrund war, dass im Verlauf der 1980er Jahre die Zahl der an Bankkrediten beteiligten Institutionen anstieg und zu einer diffuseren Gläubigerstruktur führte. Als die meisten problematischen Bankkredite in Brady-Bonds umgewandelt wurden (siehe auch Fußnote 5), konzentrierte sich die nun marktbasierte Gruppe der Gläubiger wieder (es waren vor allem große Investmentfonds, die die ersten Brady-Bonds kauften). Doch diese Konzentration war von kurzer Dauer. Als Folge der Einführung von BradyBonds legten Schwellenländer seit den frühen 1990er Jahren fast ausschließlich handelbare Staatsanleihen auf und suchten einen immer breiteren Markt für ihre Schuldentitel. Die parallel verlaufende Diversifizierung privater Anlegerportfolios mit stärkerem Gewicht auf internationale Schuldentitel (eine direkte Konsequenz der Finanzmarktglobalisierung und der neuen Technologien) führte zu einer extremen Zerstreuung der Gläubigergruppe (Enderlein 2008). Präzise Daten zur Gläubigerstruktur gibt es in keinem der veröffentlichten Datensätze. Deshalb wird auch in diesem Aufsatz die Schuldenart als „Proxy“ gewählt.

\subsection{Politisch-institutionelle Kriterien}

\subsubsection{Kooperativ vs. konfliktuell}

Wie weiter oben beschrieben, geht es bei einem Umschuldungsprozess um ein Reputationsspiel zwischen Schuldnerregierung und Gläubigern in Abwesenheit einer rechtlich übergeordneten Instanz. Ob der Umschuldungsprozess am Ende 
eher kooperativ abläuft oder konfliktuell, hängt dabei vor allem vom Verhalten der Regierung selbst ab. Enderlein et al. (2012) kodieren daher das Verhalten der Regierung sowohl was den Verhandlungs- als auch den Zahlungsprozess betrifft, um einen Indexwert für das Gesamtverhalten der Regierung zu kodieren. Der gleitende Fünf-Jahres-Durchschnittswert dieses Indizes liegt für den Zeitraum 1980-2007 stabil nah bei 2.5. Die Varianz innerhalb des Index ist dabei sehr hoch und reicht von eher kooperativen Fällen (zu denen auch die Umschuldung Griechenlands 2012 gehört [Zettelmeyer et al. 2013]) bis zu den besonders konfliktuellen Fällen, vor allem Argentinien 2002-2005. Der Kooperationsgrad der Regierung ist dabei abhängig davon, ob sie den Umschuldungsprozess von Beginn an in enger Abstimmung mit den Gläubigern durchführt oder nach einem Moratorium auf den Schuldendienst die Verhandlungen verweigert (43 Prozent der Fälle im jahresbasierten Datensatz bei Enderlein et al. 2012, wobei in nur 12 Prozent der Fälle ein echtes Moratorium ausgesprochen wird), mit einem Komplettverlust für die Anleger droht (16 Prozent der Fälle), die Datenlage verschleiert (8 Prozent der Fälle) oder auch ein Umschuldungsangebot vorlegen, das die Gläubiger nur entweder annehmen können - mit den vorgeschlagenen Verlusten - oder ablehnen mit dem Risiko, Totalverlust zu erleiden (5 Prozent der Fälle).

\subsubsection{Kurz vs. lang}

Die Dauer des Umschuldungsprozesses zwischen dem Moment des Zahlungsausfalls (oder der Ankündigung, die Schulden nicht komplett bedienen zu können) und der Annahme des Umschuldungsangebots variiert stark (Benjamin u. Wright 2009). Aus den Daten von Cruces und Trebesch (2013) ergibt sich eine durchschnittliche Dauer von rund fünf Jahren. Trebesch (2010) zeigt, dass die wichtigsten Determinanten dabei weder die Gläubigercharakteristika (s. o.) sind, noch eine diffuse Gläubigerstruktur zu längerer Verhandlungsdauer führt, sondern die politisch-institutionellen Variablen wie politisches Risiko (ICRG Index), Regierungsinstabilität oder die politische Stabilität insgesamt (basierend auf Databanks International; vgl. auch Dreher et al. 2006).

\subsubsection{Ex-ante vs. ex-post Umschuldung}

Verbunden mit den beiden vorigen Kriterien ist auch die Frage, ob eine Regierung zuerst die Zahlungen an die Gläubiger einstellt und im Anschluss die Umschuldungsverhandlungen aufnimmt (ex-post oder „post default“), oder ob sie präventiv auf ein Umschuldungsabkommen hinarbeitet, das dann direkt durchgeführt wird (ex-ante oder „pre-default“). Das et al. (2012) kommen für den Zeitraum 1950-2010 auf 109 Umschuldungen ex-post und 77 Umschuldungen ex-ante.

\subsubsection{HIPC vs. non-HIPC}

Eine Sonderstellung im Universum der Staatsbankrotte und Umschuldungen nehmen besonders arme Länder mit hohen Auslandsschulden ein, die von der Weltbank als „highly indebted poor countries“ (HIPC) eingestuft werden. Um- 
schuldungen in diesen Ländern werden in der Regel durch die Weltbank selbst kofinanziert, indem sie den Schuldnerregierungen Gelder zur Verfügung stellt, mit denen diese ihre eigenen Schulden zu einem Bruchteil des Nominalwerts zurückkaufen können (Weltbank 2007). Cruces und Trebesch (2013) identifizieren 23 Fälle von 180 im Datensatz als HICP oder „Donor-Sponsored“-Fälle. Sie zeigen, dass Schuldenschnitte bei diesen Fällen deutlich höher liegen als im Rest der Datensätze (87 Prozent vs. 30 Prozent).

Eine Sonderstellung in der Klassifizierung sollten auch sog. Brady-Bonds einnehmen. Dieses Kriterium wird hier jedoch nicht gesondert aufgeführt, weil es keiner Dynamik unterliegt, sondern ein einmaliges Regime beschreibt. ${ }^{5}$

\subsection{Rechtliche Kriterien}

\subsubsection{Rechtsstreit vs. kein Rechtsstreit}

Wie weiter unten im Detail ausgeführt wird, sind Rechtsstreite inzwischen zu einem wichtigen Instrument bei oder nach Umschuldungsprozessen geworden. Wie A3 zeigen, hat sich der dem Immunitätsgedanken zugrunde liegende Rechtsrahmen seit den späten 1970er Jahren sukzessive verändert. Dadurch ist der Anreiz für die ursprünglichen Anleihehalter oder auch spezialisierte Fonds, die von Umschuldungen betroffene Anleihen zu geringen Preisen aufkaufen (manchmal als „vulture funds“ oder „Geier-Fonds“ bezeichnet), sich auf dem Rechtsweg eine volle Auszahlung zu sichern, deutlich gestiegen. A3 identifizieren für den Zeitraum seit 1970120 Fälle, wobei der Anteil Argentiniens mit 41 Klagen besonders hoch ist.

\subsubsection{Collective Action Clauses vs. keine Collective Action Clauses}

Ein weiteres neues rechtliches Charakteristikum bei Umschuldungen ist die Präsenz von sogenannten „Collective Action Clauses“ (CAC) im Anleihevertrag (Gelpern u. Gulati 2013, IMF 2013). Solche CACs sorgen dafür, dass bei einer Umschuldung die Zustimmung eines ex-ante definierten Anteils der Gläubiger (z. B. 66\%) für alle Gläubiger bindend wird und damit keine Rechtstreite nach der Umschuldung mehr angestrengt werden können. Bislang ist der noch laufende Bestand von Staatsanleihen nur zum Teil mit CACs ausgestattet. Zudem wird bestritten, dass die Durchschlagskraft dieser CACs im Ernstfall wirken kann, weil sich gerade spezialisierte Investoren in einzelnen, eher kleinen Anleihekategorien recht leicht eine Sperrminorität sichern können.

5 Mit den „Brady-Bonds“ wurde die Staatsschuldenkrise in Lateinamerika der 1980er Jahre weitgehend beendet. In den späten 1980er Jahren befanden sich viele dieser Länder bereits seit fast einem Jahrzehnt in einer Staatsinsolvenz, die durch Fristenverlängerungen nur künstlich aufgeschoben wurde. Durch die Umschuldung in Brady-Bonds wurden weitreichende Schuldenschnitte durchgeführt, weil die Bankkredite in Anleihen mit geringerem Nominalwert umgewandelt wurden, gleichzeitig aber teilweise durch US-Anleihen abgesichert wurden (Das et al. 2012; Henry u. Arslanalp 2005, siehe auch die Kritik von Chuhan u. Sturzenegger 2005). Es gab 17 Brady-Bond Fälle. 


\subsection{Auswertung}

Die Nebeneinanderstellung dieser insgesamt elf Kriterien macht deutlich, wie differenziert Staatsbankrotte und Umschuldungsprozesse betrachtet werden müssen. Die Kausalbeziehungen zwischen den einzelnen Kriterien sind bislang nur teilweise untersucht worden. Am ausgereiftesten ist die Forschung zur Größe des Schuldenschnitts in Bezug auf andere hier vorgestellte Kriterien (siehe Cruces u. Trebesch 2013 für eine Übersicht). Aber die multiplen Kausalbeziehungen, die zwischen den einzelnen Teilkriterien bestehen, sind bislang nicht ausreichend untersucht worden und können aufgrund der letztlich eher engen Datenlage wohl auch nur begrenzt identifiziert werden. Für die politökonomische Literatur ist es jedoch bereits von zentraler Bedeutung, die breiten Dynamiken der Entwicklung von Staatsbankrotten und Umschuldungen zu erkennen. Wie der folgende Abschnitt zeigt, lassen sich anhand der hier aufgestellten elf Kriterien deutliche Trends in der Entwicklung ablesen, die auch ohne einen detaillierten Kausalzusammenhang darauf hinweisen, dass ein neues Zeitalter von Schuldenkrisen, Staatsbankrotten und Umschuldungen begonnen hat.

\section{Veränderungsprozesse seit der Mitte der 1990er Jahre: das neue Zeitalter von Schuldenkrisen}

Die im vorigen Abschnitt aufgelisteten Kriterien liegen fast komplett in den drei diesem Aufsatz zugrunde liegenden Datensätzen als Längsschnittvariablen vor. Dadurch wird es möglich, grundlegende Trends zu erkennen. Und es ist von fundamentaler Bedeutung für das Verständnis der internationalen und vergleichenden politischen Ökonomie von Schuldenkrisen und Staatsbankrotten, dass seit ca. der Mitte der 1990er Jahre in fast allen hier aufgeführten Kriterien weitereichende Veränderungsprozesse stattgefunden haben. Je nach Kriterium fallen diese Veränderungen mehr oder weniger deutlich aus, und auch der präzise Moment des „Strukturbruchs“, der je nach Variable auch quantitativ bestimmt werden könnte, ist nicht für alle Kriterien gleich. Gleichwohl sticht ein Veränderungsprozess hervor, den dieser Abschnitt beschreibt. Auch wenn eine Erklärung der Veränderungen aufgrund der hohen Anzahl von möglichen Interaktionseffekten sowohl empirisch als auch argumentativ kaum möglich ist, so wird in diesem Abschnitt doch auf eine Reihe von möglichen Kausalbeziehungen hingewiesen.

Die folgenden Unterabschnitte greifen die grobe Kriterien-Struktur des vorigen Abschnitts auf, verbleiben jedoch auf der Ebene der vier Grundkategorien (ökonomisch, finanzmarktbezogen, politisch-institutionell, rechtlich). Die Frage, warum gerade die Mitte der 1990er Jahre einen so wichtigen Einschnitt markiert, wird dann am Ende dieses Abschnitts in einer Gesamtschau behandelt.

\subsection{Häufigkeit und ökonomische Kriterien}

Die Häufigkeit von Staatsbankrotten hat sich in den vergangenen 40 Jahren fundamental verändert. Während es im Zeitraum 1980-1995 im Durchschnitt zu fast 14 Staatsbankrotten pro Jahr kam, waren es im Jahrzehnt 1996-2006 nur noch knapp drei (Enderlein et al. 2012; siehe auch Abbildung 4). 
Es ist jedoch bemerkenswert, dass sich die ökonomischen Kriterien im gleichen Zeitraum fundamental verschoben haben. Abbildung 2 zeigt einen deutlichen Trend seit den frühen 1980er Jahren: nicht nur bei der reinen Höhe der „Haircuts“, sondern vor allem unter Einbeziehung der Volumina. Gerade die großen Umschuldungen führen zu großen Schuldenschnitten. Die großen Umschuldungen der vergangenen Jahre, nämlich Griechenland 2012, Argentinien 2005, Russland 2000 und Irak 2006, weisen alle Schuldenschnitte von mehr als 50 Prozent auf. Insgesamt waren die durchschnittlichen Kapitalwertverluste in den 1990er und 2000er Jahren um 25 Prozentpunkte höher als in den 1980er Jahren (Cruces u. Trebesch 2013).

\section{Abbildung 2: Volumina und „Haircuts“}

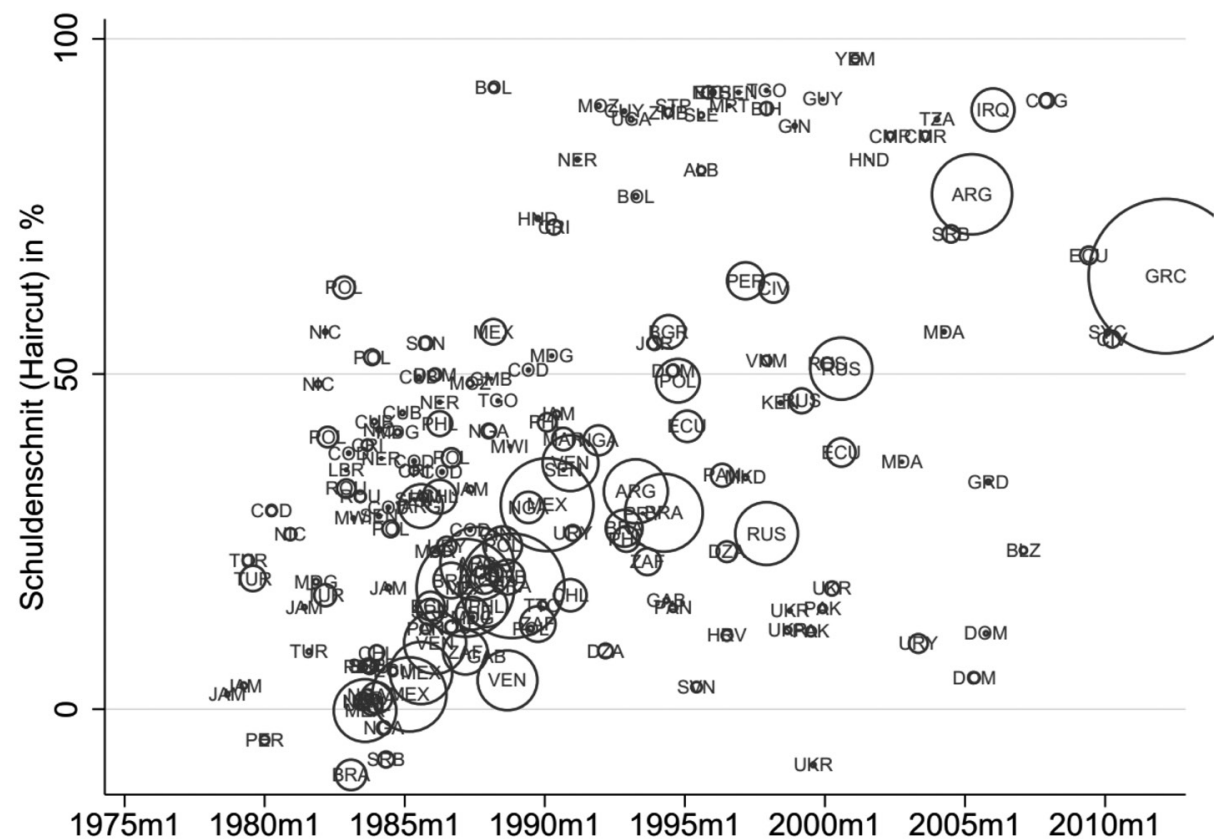

Quelle: Eigene Darstellung auf Grundlage von Cruces u. Trebesch 2013, sowie Zettelmeyer et al. 2013

Auch die Art des Schuldenschnitts veränderte sich im gleichen Zeitraum. Wie Abbildung 2 deutlich zeigt, wiesen die Umschuldungen der 1980er Jahre keine Schuldenschnitte auf, sondern in der Regel Fristenverlängerungen. Seit den 1990er Jahren waren es aber gerade die Umschuldungen mit hohem Kapitalwertverlust oder „Haircut“, die eine Entlastung vor allem über eine direkte Reduzierung des Nominalwerts der Schulden erreichten. Die 57 Fälle, bei denen auch der „face value“ der Anleihe reduziert wurde (und die fast alle nach 1990 auftreten), weisen einen durchschnittlichen Kapitalwertverlust von fast 65 Prozent auf, wohingegen die 123 Fälle der Fristenverlängerungen nur auf gut 24 Prozent kommen (Cruces u. Trebesch 2013). Zu einem gewissen Grad ist dieser Zusammenhang 
logisch, weil höhere Haircuts nur über extrem lange Fristenverlängerungen zu extrem niedrigen Zinsen erreicht werden können oder eben durch eine Reduktion des Nominalwerts. Dennoch wird deutlich, wie sehr die Schuldentragfähigkeit seit der Mitte der 1990er Jahre in den Vordergrund der Schuldendebatte gerückt ist.

Abbildung 3: Fristenverlängerungen vs. Schnitt auf Nominalwert und „Haircuts“

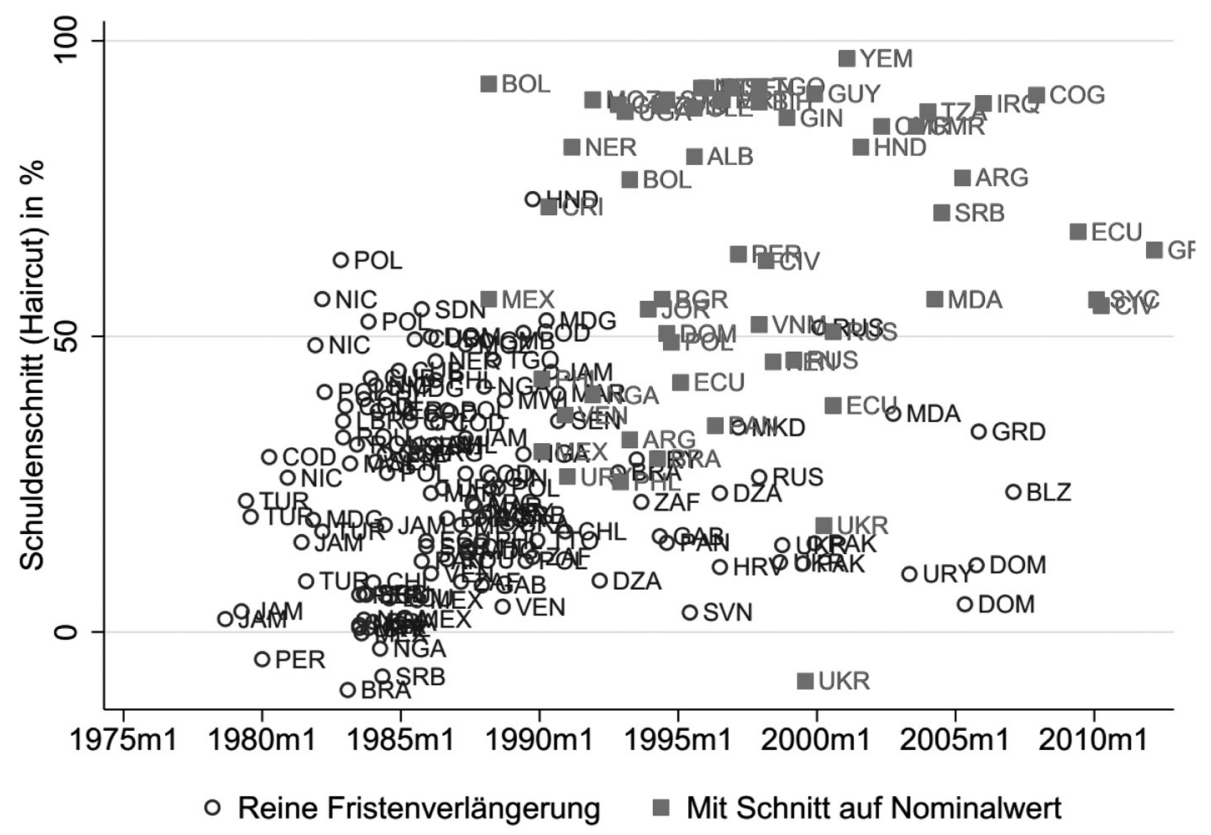

Quelle: Eigene Darstellung auf Grundlage von Cruces u. Trebesch 2013, sowie Zettelmeyer et al. 2013

\subsection{Finanzmarktkriterien}

Zuerst ist aus dem vorigen Unterabschnitt festzuhalten, dass sich die Zeit seit der Mitte der 1990er Jahren anhand einer Kombination aus selteneren Umschuldungen, dafür dann aber mit höheren „Haircuts“ und großen Volumina charakterisieren lässt. Diese Entwicklung verdient Beachtung, weil sie in direktem Zusammenhang gestellt werden kann mit der größeren direkten Verbindung zwischen den nationalen Finanzmärkten im globalen System: Die finanzielle Globalisierung könnte ein Erklärungsfaktor dafür sein, dass Umschuldungen generell seltener geworden sind, weil die Reputations- und auch systemischen Kosten deutlich gestiegen sind; wenn sie aber durchgeführt werden müssen, dann geschieht dies im großen Stil. ${ }^{6}$

6 Unter Umständen könnten die gestiegenen Kosten dazu führen, dass Umschuldungen zu einem ineffizient späten Zeitpunkt durchgeführt werden (Bolton u. Jeanne 2007, 2009). 
Dazu passt, dass der Anteil von Anleiheumschuldungen seit Mitte der 1990er Jahren durch die Entstehung des internationalen Anleihemarktes deutlich angestiegen ist. Zwar ist die Gesamtanzahl mit 22 Anleiheumschuldungen weiter sehr gering, doch das durchschnittliche Umschuldungsvolumen dieser Fälle ist mit ca. 17.5 Milliarden US Dollar mehr als dreimal so hoch wie der Durchschnittswert des gesamten Datensatzes (Cruces u. Trebesch 2013).

Bemerkenswert ist jedoch, dass die Gläubigerstrukturen, die in der Literatur und in der Politik ex-ante oft als zentraler Auslöser eines Strukturwandels bei Umschuldungen bezeichnet wurden und eine Debatte über einen „Sovereign Debt Insolvency Mechanism“ (SDRM) auslösten, am Ende kaum ins Gewicht fielen (siehe hierzu die Ausführungen in Panizza et al. 2009, S. 671 und insbesondere die Beiträge zur Gläubigerstruktur und der Debatte über den SDRM von Eichengreen u. Portes 1995; Kruger u. Haldane 2001; Rogoff und Zettelmeyer 2002; Eichengreen et al. 2003; Bi et al. 2011; Moody's 2013). Die Gläubigerstruktur scheint nicht für die Dauer von Umschuldungen ausschlaggebend zu sein (Trebesch 2010).

\subsection{Politisch-institutionelle Kriterien}

Abbildung 4 zeigt, dass sich der durchschnittliche Kooperationsgrad von Regierungen in Umschuldungsprozessen trotz aller anderen Veränderungsdynamiken seit der Mitte der 1990er Jahren, insbesondere was die Häufigkeit der Umschuldungen betrifft, kaum signifikant verschoben hat. Zwar schlägt der Index von Enderlein et al. (2012) wegen der geringeren Fallzahlen massiver aus, doch der Fünf-Jahres-Durchschnittswert bleibt stabil.

Eine mögliche Erklärung dieser Stabilität ist die stärkere Polarisierung in den Umschuldungsfällen, die auch schon in den beiden vorigen Unterabschnitten deutlich wurde. Wenn es zu Umschuldungen kommt, dann fallen diese groß aus, sowohl beim Schuldenschnitt als auch beim Volumen. Gleichzeitig können diese Umschuldungen dann entweder besonders kooperativ gelöst werden (wie in den Fällen Uruguay 2001 und Griechenland 2012) oder besonders konfliktuell (wie im Fall Argentiniens 2005). 


\section{Abbildung 4: Anzahl Staatsbankrotte und Kooperationsgrad}

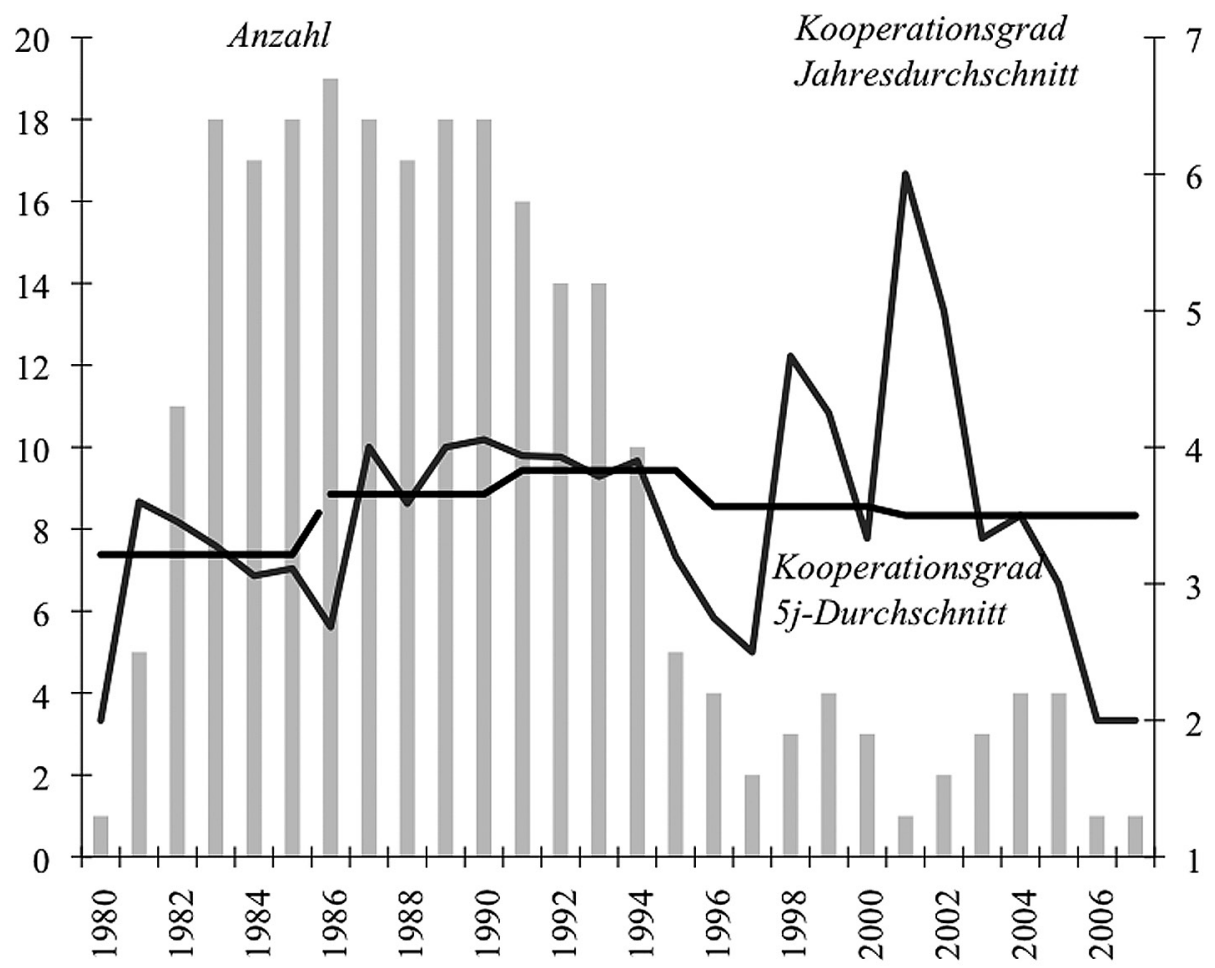

Quelle: Enderlein et al. 2012

Auch die Dauer der Krisen hat sich fundamental verändert. Die Umschuldungen der 1980er und 1990er Jahre dauerten im Durchschnitt 31 Monate, während sie nach 1998 nur noch 17 Monate dauerten (Das et al. 2012, S. 27). Dabei ist auch hier der Trend zur Polarisierung bemerkenswert: Während der besonders konfliktuelle Fall Argentiniens 2002-2005 drei Jahre dauerte, wurden viele andere Fälle innerhalb von nur wenigen Monaten ausgehandelt.

\subsection{Rechtliche Kriterien}

Besonders fundamental hat sich seit Mitte der 1990er Jahre allerdings das Rechtsumfeld verändert. Es könnte diese Veränderung sein, die mit besonderem Gewicht in die zukünftige Analyse von Staatsbankrotten einfließen wird. Denn in der Tat haben mehrere Gerichtsverfahren in den USA und Großbritannien seit der Mitte der 1970er Jahre den besonderen Schutz staatlicher Aktiva aufgrund der Immunität immer stärker eingeschränkt. Zwar gilt die Grundidee der Immunität weiter, doch vor allem durch die Fälle Weltover vs. Argentina (1992, Southern District of New York, 89 Civ. 6926) und Elliot vs. Peru (2000, Southern District of New York, 98 Civ. 8681) wurden Staatsanleihen privaten Anleihen in der Rechtsbetrachtung stär- 
ker gleichgestellt und zudem bestimmte Anleiheklauseln dergestalt ausgelegt, dass alle Anleihehalter grundsätzlich gleich zu behandeln seien („pari passu“). Der Staatsbankrott Argentiniens 2001 und die schwierige Umschuldung, die dann 2005 erfolgte, lösten zahlreiche Rechtsstreite aus, insbesondere den inzwischen weiteren ganz besonderen Fall NML Capital vs. Argentina (2011-2014, Southern District of New York, 08 Civ. 6978), den ein New Yorker Gericht dahingehend entschied, dass Argentinien die Anleihehalter, die dem Umschuldungsabkommen zugestimmt hatten, nur dann weiter durch Zahlungen bedienen darf, wenn auch NML (ein zum großen Hedge Fonds Elliott Management gehörender sog. „Holdout“-Gläubiger, also ein willentlich nicht an der Umschuldung teilnehmender Halter der alten Anleihen) in seinen ursprünglichen Ansprüchen ebenfalls vollständig bedient würde. Das US-Gericht drohte an, US-Banken, die Argentiniens Zahlungen weiterleiteten, unter dem Aspekt der Beihilfe zum Vertragsbruch zu belangen.

Dieser sehr prominente Fall ist aber nur ein Beispiel des deutlichen Anstiegs von Anlegerklagen in den vergangenen 25 Jahren. Abbildung 5 zeigt den deutlichen Trend sowohl bei den Volumina als auch bei der Anzahl der Fälle (Schumacher et al. 2014). Inzwischen ist jede zweite Umschuldung von einer Anlegerklage betroffen. Ähnlich zu den Entwicklungen des „Junk Bond“-Markts für in Zahlungsschwierigkeiten steckende Unternehmen hat sich inzwischen ein Zweig der Hedge Fonds-Branche auf den Ankauf von umgeschuldeten Anleihen „im Bankrott" spezialisiert (sog. „vulture funds“ oder „Geier-Fonds“), der diese zu sehr geringen Preisen aufkauft, um im Anschluss seine Ansprüche durch die Androhung oder tatsächliche Anwendung des Rechtswegs geltend zu machen.

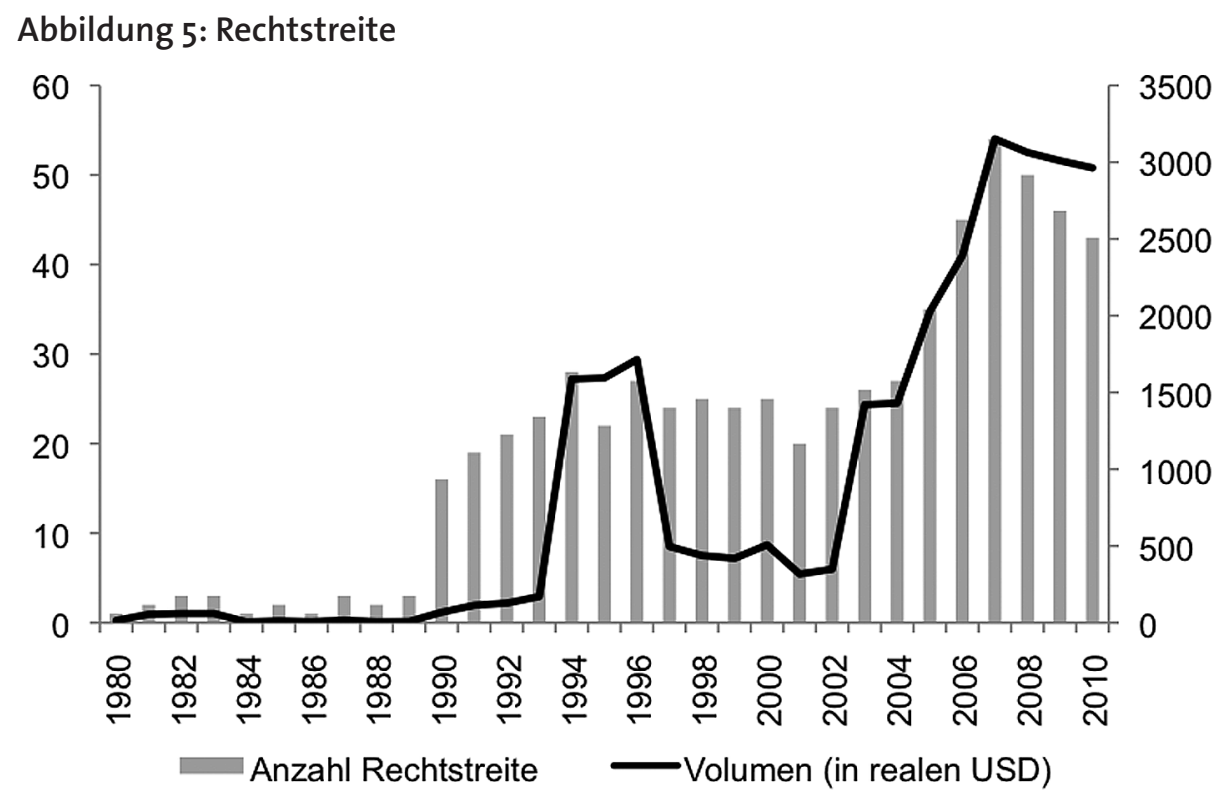

Quelle: Schumacher et al. 2014 
Diese Entwicklung ist für den internationalen Anleihemarkt von herausragender Bedeutung. Denn die bislang sowohl politisch als auch wissenschaftlich oftmals formulierte Grundannahme, dass der Markt für Staatschulden einer reinen Reputationslogik zwischen Staaten und Privatgläubigern gehorcht, würde durch eine immer größer werdende Welle von Anlegerklagen grundsätzlich in Frage gestellt. Schon am Fall Griechenlands lässt sich ablesen, welche Implikationen diese Entwicklung haben könnte. Während die Umschuldung Griechenlands 2012 durch eine unilaterale Veränderung der unter griechischem Recht begebenen Anleiheverträge durch das griechische Parlament beschlossen wurde und Anlegerklagen in Griechenland damit kaum erfolgsversprechend waren, galt bei den griechischen Anleihen nach ausländischem Recht (z. B. Schweizer Recht, britisches Recht) eine ganz andere Logik: Dei diesen Anleihen entschied sich die griechische Regierung, den Holdout-Gläubigern keinen Schuldenschnitt aufzuzwingen, falls diese dem Umtausch nicht freiwillig zustimmten, weil sie Anlegerklagen nach britischem oder Schweizer Recht fürchtete (Zettelmeyer u. Gulati 2012). Diese Implikation deutet darauf hin, dass Staatsbankrotte in naher Zukunft zwar immer seltener werden, dann aber lange Rechtsstreitigkeiten nach sich ziehen könnten.

Aber auch der Trend zu einer juristischen Auseinandersetzung über Staatsschulden kann nicht losgelöst von den anderen hier betrachteten Kriterien analysiert werden. Wie weiter oben angedeutet, hat sowohl das veränderte Finanzmarktumfeld als auch der veränderte politische Kontext dazu beigetragen, dass Anlegerklagen zugenommen haben. Panizza et al. weisen auf einen Zusammenhang zwischen konfliktuellem Regierungsverhalten in Umschuldungsverhandlungen und der Höhe von Haircuts hin (2009), wobei die Höhe des Haircuts der wichtigste Erklärungsfaktor von Gläubigerklagen in neueren Anleihe-Umschuldungen ist.

In einer Gesamtschau lässt sich folgende Tabelle aufstellen, die die elf Kriterien zusammenfassend aufbereitet und die wichtigsten Trends darstellt. 


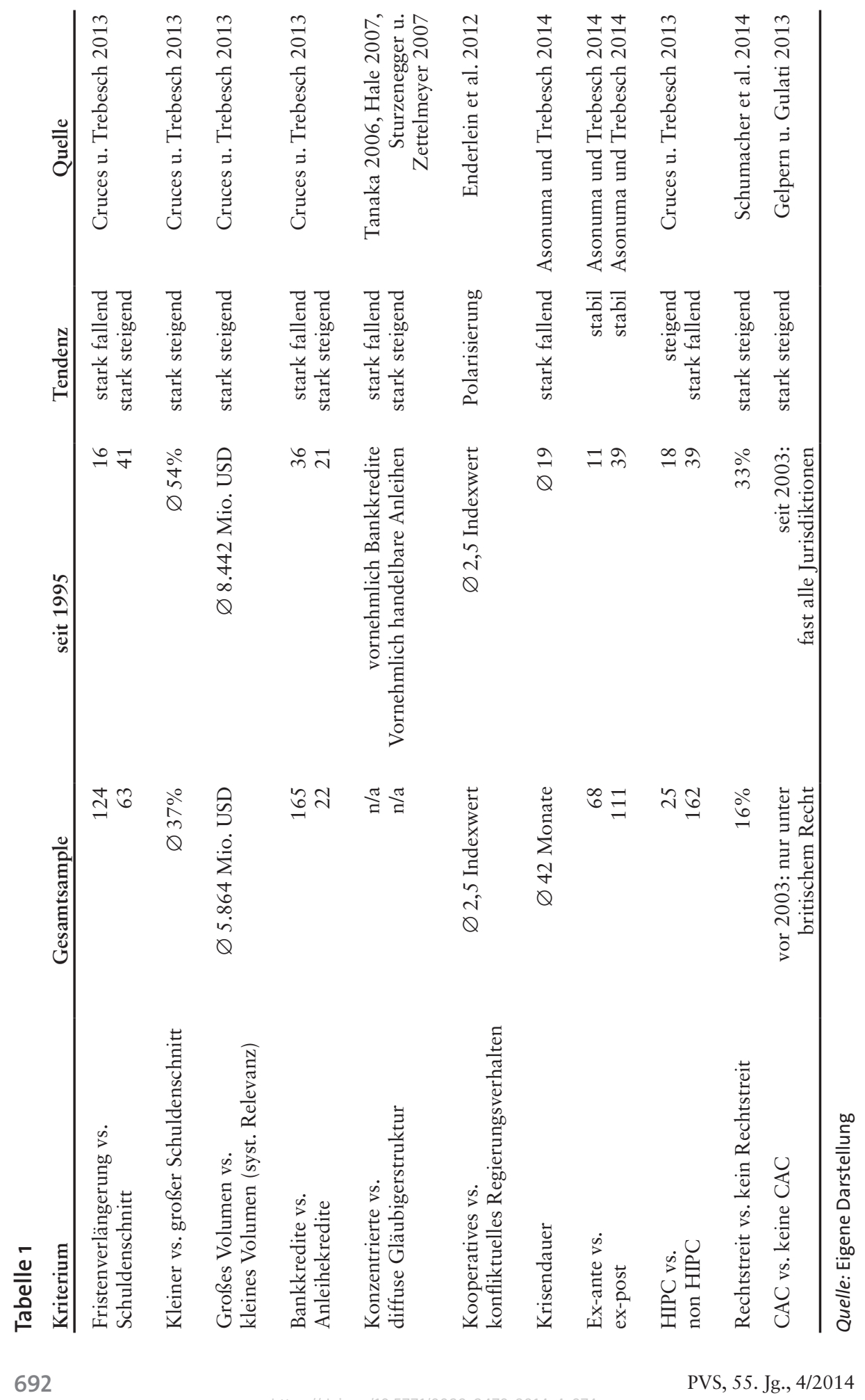




\section{Schlussfolgerungen für die Politikwissenschaft und die politische Ökonomie}

Die Kernfrage der politikwissenschaftlichen Auseinandersetzung mit Staatsbankrotten und Umschuldungen bleibt weiter, warum Gläubiger Regierungen ausreichend Vertrauen schenken, wenn sie keine direkte Rechtsdurchsetzungsmöglichkeit haben, um im Zahlungsausfall auf Sicherheiten zugreifen zu können. Die wichtigste Antwort liegt in der Reputation des Staates, die sich durch hohe „Haircuts“ in der Umschuldung verschlechtert und somit den Zugang zum Anleihemarkt in den Folgejahren nach einem Staatsbankrott im Durchschnitt deutlich erschwert (Cruces und Trebesch 2013). Diese Marktlogik ist theoretisch solide untermauert und die empirischen Ergebnisse sind insgesamt signifikant, doch es gibt ausreichend Gegenbeispiele oder Ausnahmen, die zeigen, dass weitere Forschung dringend nötig ist.

Aus politökonomischer Sicht wirft der Reputationsansatz insgesamt mehr Fragen auf, als er beantwortet. Seine argumentative Grundlage ist ein langer Zeithorizont des Schuldnerlandes. Er nimmt an, dass Regierungen in der Lage sind, „deferred gratifications“ (Elster 1979) zu erkennen und als Handlungsgrundlage zu akzeptieren. Parallel dazu geht dieser Ansatz davon aus, dass Finanzmärkte nicht Regierungen als ihre Schuldner betrachten, sondern die Länder als Einheiten. Es ist für eine Finanzierungsentscheidung nicht erheblich, welche Regierung sich an der Macht befindet.

Diese Annahmen blenden aber sowohl den Zeithorizont als auch den institutionellen Handlungskontext der Schuldnerregierung aus. Wenn Staatsverschuldung als apolitische Komponente der Regierungshandlung aufgefasst werden könnte, dann wäre ein solches Vorgehen gerechtfertigt: Das Schuldenproblem wäre ein Länderproblem, kein Regierungsproblem, und bei der Reputationsproblematik würde es um Länderreputation gehen, nicht um Regierungsreputation.

Vor diesem Hintergrund weist der Aufsatz darauf hin, warum bei der Analyse von Staatsbankrotten eine erhöhte Differenzierung zwischen den Einzelfällen unabdingbar ist. Der Mangel daran könnte erklären, warum die politökonomische Literatur zu Schuldenkrisen zu so teils widersprüchlichen Ergebnissen kommt. So bleiben die wichtigsten in der Einleitung aufgeworfenen theoretischen Fragen entweder unbeantwortet oder werden widersprüchlich beantwortet.

Dieser Aufsatz und seine zugrunde liegende Forschungsarbeit haben es sich deshalb zum Ziel gesetzt, die abhängigen Variablen in der relevanten Literatur, also die Typen der Umschuldungen und Staatsbankrotte, mit ihren wichtigsten Charakteristika herauszuarbeiten und für die Forschung verwendbar zu machen. Der Aufsatz hat auch gezeigt, dass diese Charakteristika untereinander teilweise korrelieren und Kausalbeziehungen keineswegs ausgeschlossen sind. Doch die Komplexität der möglichen Interaktionseffekte bei gleichzeitig sehr geringer empirischer Datenbasis macht die Forschung so schwierig. Die politökonomische Forschung hat drei nicht wirklich zufriedenstellenden Strategieoptionen, um auf diese Herausforderung zu reagieren. Erstens die starke Komplexitätsreduktion, die, um validierbare empirische Ergebnisse zu erzielen, völlig unvergleichbare Staatsbankrott-Fälle als identisch deklarieren muss, selbst wenn diese - wie unser Aufsatz gezeigt hat - für sehr unterschiedliche politökonomische Phänomene stehen. Zweitens die Rückkehr zu Fallstudien und Process-Tracing-Methoden, die zwar die Eigenheiten der Fälle berücksichtigen können, am Ende aber immer un- 
ter den Schwächen bei der allgemeinen Validierbarkeit von Ergebnissen leiden. Drittens die rein theoretische Arbeit, die ganz auf Empirie verzichtet und in der Tradition der ursprünglichen Literatur zu Staatsbankrotten auf grundlegende Interaktionsmechanismen hinweist, ohne diese wirklich testen zu können.

Letztlich bleibt wohl die Kombination aller drei Vorgehensweisen. Aber die in diesem Aufsatz vorgenommene Zerlegung des Staatsbankrottkonzepts zeigt, welche forschungsstrategischen Herausforderungen mit dem Thema verbunden sind.

Von zentraler Bedeutung für die politökonomische Forschung ist das Thema allemal. Denn im zurückliegenden Jahrzehnt haben sich Staatsbankrotte und Umschuldungen als wichtiger Bestandteil der wirtschaftspolitischen Praxis festgesetzt. Die historisch hohen Schuldenstände werden oft als Zeichen dafür gewertet, dass weitere Umschuldungen zu erwarten sind (Reinhart u. Rogoff 2009).

Sollte dieses Szenario eintreten, dann - so zeigen wir in diesem Aufsatz - könnten politisch-institutionellen Variablen eine erhebliche Bedeutung zufallen. Denn auch wenn die Häufigkeit von Umschuldungen im vergangenen Jahrzehent zurückgegangen ist, so handelt es sich bei den wenigen Umschuldungen oft um besonders relevante Fälle, entweder mit sehr hohen Schuldenschnitten, hohen Volumina, einem hohen Konfliktgrad und einem Trend zur Gläubigerklage (Beispiel: Argentinien 2005) oder hohen Schuldenschnitten bei hohen Volumina, einem sehr viel geringeren Konfliktgrad und wenigen Gläubigerklagen (Beispiel: Griechenland 2012).

Idealtypisch lässt sich aus den hier vorgestellten Datensätzen ableiten, dass Umschuldungen in Zukunft nach zwei groben Szenarien ablaufen könnten, die fast schon auf eine binäre oder polarisierende Entwicklung in der politischen Ökonomie von Staatsbankrotten hindeuten.

Ein Szenario würde zu Regierungen passen, die unter starkem ökonomischen Druck stehen, die politischen Kosten der Konsolidierung aber so hoch einschätzen, dass sie durch einen Schuldenschnitt zumindest einen Teil der Konsolidierung auf die Gläubiger abwälzen wollen. Anstatt den Schuldendienst aber komplett aufzukündigen, würden solche Regierungen eine „defensive“ Umschuldung einleiten, die auf frühe Verhandlungen und Kooperation setzt, Gläubigerklagen weitestgehend durch Klauseln wie CACs zu erschweren versucht, sich am Ende zwar eines substanziellen Teils der Staatsschulden durch große „Haircuts“ entledigt, den Gläubigern im Gegenzug aber noch verhältnismäßig attraktive Konditionen im Schuldentausch anbietet, ggf. unterstützt durch internationale Organisationen wie den IWF oder die EU. Würde sich die Welt auf solche Fälle beschränken, dann könnte zur Begleitung eines solchen kooperativen Prozesses, der sich an den Regeln für „gutes“ oder "faires" Schuldnerverhalten anlehnt (wie sie der Internationale Währungsfonds oder auch das Institute for International Finance, der Lobbyverband internationaler Großbanken, befürworten [IWF 1999, 2002 und IIF 2013]), auch ein stärker institutionell eingebetteter Prozess für Umschuldungen aufgebaut werden.

Das zweite Szenario hingegen ist ein deutlich schwierigeres, in dem eine Regierung unter starkem ökonomischem Druck die politischen Kosten der Konsolidierung schlicht ablehnt. Gerade im Anschluss an Wahlen, wenn eine Regierung gegen die Konsolidierung Wahlkampf geführt hat, wäre eine offensive Umschuldung denkbar; also eine sehr direkte Verkündung eines Schuldenmoratoriums mit dem Ziel, dem Land größtmöglichen kurzfristigen finanziellen Handlungsspielraum zu 
verschaffen, der politische Stabilität und Wachstum bringen könnte. Diese Strategie könnte sich auf die Vermutung stützen, dass eine erfolgreiche „offensive“ Umschuldung dieser Art schnelle ökonomische Erfolge bringen könnte und damit der Marktzugang trotz des entstandenen Reputationsverlusts schon recht kurze Zeit nach der Umschuldung wieder möglich wäre. Gläubigerklagen spielen in diesem Szenario eine weitaus bedeutendere Rolle, weil sich ggf. nur wenige Gläubiger einer freiwilligen Umschuldung anschließen würden. Das letztliche Ergebnis des Rechtsstreits (und der Verhandlungen) zwischen Argentinien und dem „GeierFonds" Elliott könnte die strategischen Optionen von Regierungen in Staatsbankrotten auf Jahrzehnte hin neu ausrichten. Sollte sich Argentinien trotz der Zahlungsverpflichtung durch ein US-Gericht am Ende dieser Auflage entziehen können, dann wäre dies ein Zeichen für viele Nationalstaaten, dass die Zeit unilateraler und konfliktueller Umschuldungen noch nicht vorüber ist.

Für die internationale politische Ökonomie ist von Bedeutung, welche Hierarchie- und Governance-Strukturen die globale Schuldenproblematik bei geringstmöglichen negativen Externalitäten in den Griff bekommen könnten. Die recht erfolgreichen HIPC Umschuldungen seit den frühen 1990er Jahren sind ein Beispiel dafür, dass internationale Kooperation auch bei direkten finanziellen Interessen möglich ist. Die erheblichen politischen Konflikte um den griechischen Staatsbankrott in Kombination mit einem hohen Betrag an neuem Geld durch die Mitgliedsländer der europäischen Währungsunion und den Internationalen Währungsfond haben gezeigt, dass bei größeren Umschuldungen die politischen Effekte gerade aufgrund des hohen Grads an möglicher Redistribution über Grenzen hinweg nicht zu unterschätzen sein sollten.

\section{Literatur}

Archer, Candace C., Glen Biglaiser, und Karl DeRouen Jr. 2007. Sovereign Bonds and the "Democratic Advantage": Does Regime Type Affect Credit Rating Agency Ratings in the Developing World? International Organization 61: 341-365. DOI: 10.1017/ S0020818307070129.

Arellano, Cristina, Xavier Mateos-Planas, und Jose-Victor Rios-Rull. 2013. Partial Default. Mimeo. Minneapolis: Federal Reserve Bank of Minneapolis.

Benjamin, David, und Mark L.J. Wright. 2009. Recovery Before Redemption? A Theory of Delays in Sovereign Debt Renegotiations. Unveröffentlichtes Manuskript.

Bi, Ran, Marcos Chamon, und Jeromin Zettelmeyer. 2011. The Problem that Wasn't: Coordination Failures in Sovereign Debt Restructurings. IMF Working Paper No. 11/265. Washington, D. C.: International Monetary Fund.

Biglaiser, Glen, und Joseph L. Staats. 2012. Finding the "Democratic Advantage" in Sovereign Bond Ratings: The Importance of Strong Courts, Property Rights Protection, and the Rule of Law. International Organization 66: 515-535. DOI: 10.1017/ S0020818312000185.

Biglaiser, Glenn, und Karl DeRouen. 2007. Sovereign Bond Ratings and Neoliberalism in Latin America. International Studies Quarterly 51: 121-138.

BILD. 2010. Verkauft doch eure Inseln, ihr Pleite-Griechen ... und die Akropolis gleich mit! http://www.bild.de/politik/wirtschaft/griechenland-krise/regierung-athen-sparenverkauft-inseln-pleite-akropolis-11692338.bild.html. Zugegriffen: 04.09.2014 
Bolton, Patrick, und Olivier Jeanne. 2007. Structuring and restructuring sovereign debt: The role of a bankruptcy regime. Journal of Political Economy 115: 901-924.

Bolton, Patrick, und Olivier Jeanne. 2009. Structuring and restructuring sovereign debt: The role of seniority. The Review of Economic Studies 76: 879-902.

Bulow, Jeremy I., und Kenneth Rogoff. 1989. A Constant Recontracting Model of Sovereign Debt. Journal of Political Economy 97: 155-178.

Chuhan, Punam, und Federico Sturzenegger. 2005. Defaults Episodes in the 1980s and 1990s: What Have We Learned? In Managing Economic Volatility and Crises, Hrsg. Joshua Aizenman und Brian Pinto, 471-520. Cambridge: Cambridge University Press.

Cordes, Till. 2014. The Political Economy of Sovereign Rating Criteria. What Rating Agencies Demand from National Governments. Dissertationsschrift eingereicht an der Berlin Graduate School for Transnational Studies.

Cruces, Juan J., und Christoph Trebesch. 2013. Sovereign Defaults: The Price of Haircuts. American Economic Journal: Macroeconomics 5: 85-117. DOI: 10.1257/mac.5.3.85.

Das, Udaibir S., Michael G. Papaioannou, und Christoph Trebesch. 2012. Sovereign Debt Restructurings 1950-2010: Literature Survey, Data, and Stylized Facts. IMF Working Paper WP/12/203. Washington, D. C.: International Monetary Fund.

Dias, Daniel A., Christine Richmond, und Mark Wright. 2012. In for a Penny, In for 100 Billion Pounds: Quantifying the Welfare Benefits from Debt Forgiveness. Mimeo. Illinois: University of Illinois.

Dias, Daniel A., Christine Richmond, und Mark Wright. 2014. The stock of external sovereign debt: Can we take the data at 'face value'? Journal of International Economics 94: 1-17. DOI: $10.3386 / \mathrm{w} 17551$.

Dreher, Axel, Bernhard Herz, und Volker Carb. 2006. Is there a Casual Link between Currency and Debt Crises? International Journal of Finance and Economics 11: 305-325.

Eaton, Jonathan, und Mark Gersovitz. 1981. Debt with Potential Repudiation: Theoretical and Empirical Analysis. Review of Economic Studies 48: 289-309.

Eichengreen, Barry, und Richard Portes. 1995. Crisis? What Crisis? Orderly Workouts for Sovereign Debtors. London: Center of Economic Policy Research.

Eichengreen, Barry, Ashoka Mody, und Kenneth Kletzer. 2003. Crisis Resolution: Next Steps. NBER Working Paper No. 10095. Cambridge, Mass.: The National Bureau of Economic Research.

Elster, John. 1979. Ulysses and the Sirens. Cambridge: Cambridge University Press.

Enderlein, Henrik, Christoph Trebesch, und Laura von Daniels. 2012. Sovereign debt disputes: A database on government coerciveness during debt crises. Journal of International Money and Finance 31: 250-266. DOI: 10.1016/j.jimonfin.2011.11.011.

Enderlein, Henrik. 2008. Makroökonomische Stabilisierung in Schuldenkrisen: Zur Bedeutung von Governance Aspekten in der Interaktion von privaten Gläubigern und staatlichen Schuldnern in Schwellenländern. In Regieren obne Staat? Governance in Räumen begrenzter Staatlichkeit (Schriften zur Governance-Forschung, Band 10), Hrsg. Thomas Risse und Ursula Lehmkuhl, 309-331. Baden-Baden: Nomos Verlag.

Fearon, James. 1994. Domestic Political Audiences and the Escalation of International Disputes. American Political Science Review 88: 577-592.

Gelpern, Anna, und Mitu Gulati. 2013. The wonder-clause. Journal of Comparative Economics 41: 367-385.

Gennaioli, Nicola, Alberto Martin, und Stefano Rossi. 2014. Sovereign default, domestic banks, and financial institutions. Journal of Finance 69: 819-866. DOI: 10.1111/ jofi.12124. 
Grossman, Herschel I., und John B. Van Huyck. 1985. Sovereign debt as a contingent claim: Excusable default, repudiation, and reputation. American Economic Review 78: 1088-1097. DOI: 10.3386/w1673.

Hale, Galina. 2007: Bonds or Loans? The Effect of Macroeconomic Fundamentals. Economic Journal 117: 196-215.

Henry, Peter Blair, und Serkan Arslanalp. 2005. Is Debt Relief Efficient? Journal of Finance, American Finance Association 60: 1017-1051.

Institute of International Finance (IIF). 2013. Principles for stable capital flows and fair debt restructuring: Report on implementation by the Principles Consultative Group. Washington, D. C.: Institute of International Finance.

International Monetary Fund (IMF). 1999. IMF Policy on Lending into Arrears to Private Creditors. Washington D. C.: International Monetary Fund.

International Monetary Fund (IMF). 2002. Fund Policy on Lending into Arrears to Private Creditors: Further Consideration of the Good Faith Criterion. Washington D. C.: International Monetary Fund.

International Monetary Fund (IMF). 2013. Sovereign Debt Restructuring. Recent Developments and Implications for the Fund's Legal and Policy Framework. Washington D. C: International Monetary Fund.

International Monetary Fund (IMF). 2014. The Fund's lending framework and sovereign debt - Preliminary considerations. Washington, D. C.: International Monetary Fund.

Kruger, Mark, und Andy G. Haldane. 2001. The Resolution of International Financial Crises: Private Finance and Public Funds. Bank of Canada Working Paper 01 (20). Ottawa: Bank of Canada.

Mendoza, Enrique G., und Vivian Z. Yue. 2012. A General Equilibrium Model of Sovereign Default and Business Cycles. The Quarterly Journal of Economics, Oxford University Press 127: 889-946.

Moody's. 2013. The Role of Holdout Creditors and CACs in Sovereign Debt Restructurings. New York: Moody's.

Panizza, Ugo, Federico Sturzenegger, und Jeromin Zettelmeyer. 2009. The Economics and Law of Sovereign Debt and Default. Journal of Economic Literature 47: 651-698. DOI: $10.1257 /$ jel.47.3.651.

Ponticelli, Jacopo, und Hans-Joachim Voth. 2011. Austerity and Anarchy: Budget Cuts and Social Unrest in Europe, 1919-2008. CEPR Discussion Paper 8513. London: Centre for Economic Policy Research.

Mitchener, Kris James, und Marc D. Weidenmier. 2005. Supersanctions and Sovereign Debt Repayment. NBER Working Papers 11472. Cambridge, Mass.: The National Bureau of Economic Research.

North, Douglass C., und Barry R. Weingast. 1989. Constitutions and Commitment: The Evolution of Institutions Governing Public Choice in Seventeenth-Century England. Journal of Economic History 49: 803-832.

Reinhart, Carmen M., und Kenneth S. Rogoff. 2009. This Time is Different: Eight Centuries of Financial Folly. Princeton: Princeton University Press.

Rogoff, Kenneth, und Jeromin Zettelmeyer. 2002. Bankruptcy procedures for sovereigns: A history of ideas, 1976-2001. IMF Staff Papers Vol. 49, No. 3. Washington, D. C.: International Monetary Fund.

Schultz, Kenneth A. 2001. Looking for Audience Costs. Journal of Conflict Resolution 4: 32-60. DOI: 10.1177/0022002701045001002. 
Schultz, Kenneth A., und Barry R. Weingast. 2003. The Democratic Advantage: Institutional Foundations of Financial Power in International Competition. International Organization 57: 3-42. DOI: 10.1017/S0020818303571065.

Schumacher, Julian, Christoph Trebesch, und Henrik Enderlein. 2014. Sovereign Defaults in Court: The Rise of Creditor Litigation 1976-2010. Unveröffentlichtes Manuskript.

Sturzenegger, Federico, und Jeromin Zettelmeyer. 2006. Debt Defaults and Lessons from a Decade of Crises. Cambridge, MA: MIT Press.

Tanaka, Misa. 2006. Bank loans versus bond finance: Implications for sovereign debtors. The Economic Journal 116: 149-171

Tomz Michael. 2004. Interests, Information, and the Domestic Politics of International Agreements. Unveröffentlichtes Manuskript.

Tomz, Michael. 2007. Reputation and International Cooperation: Sovereign Debt across Three Centuries. Princeton, NJ: Princeton University Press.

Trebesch, Christoph. 2010. Delays in Sovereign Debt Restructurings. Unveröffentlichtes Manuskript.

Trebesch, Christoph, Udaibir S. Das, und Michael G. Papaioannou. 2010. Sovereign Default Risk and Private Sector Access to Capital in Emerging Markets. Chapters in Economics 20024, University of Munich: Department of Economics.

Weltbank. 2007. Debt Reduction Facility for IDA-Only Countries: Progress Update and Proposed Extension. Board Report 39310. Washington D. C.: World Bank.

Zettelmeyer, Jeromin, und Mitu Gulati. 2012. Making a Voluntary Greek Debt Exchange Work. CEPR Discussion Papers 8754. London: Centre for Economic Policy Research.

Zettelmeyer, Jeromin, und Christoph Trebesch, Mitu Gulati. 2013. The Greek debt restructuring: an autopsy. Economic Policy 28: 513-563. DOI: 10.1111/1468-0327.12014.

\section{Autorenangaben}

Prof. Dr. Henrik Enderlein, Hertie School of Governance, Jacques Delors Institut Berlin, Friedrichstrasse 180,

10177 Berlin, enderlein@hertie-school.org

Julian Schumacher, M. Sc.

Johannes Gutenberg University Mainz,

Department of Law, Management and Economics,

Jakob Welder-Weg 4,

55099 Mainz,

j.schumacher@uni-mainz.de

Prof. Dr. Christoph Trebesch

Ludwig-Maximilians-Universität München,

Schackstr. 4 / I,

80539 München,

christoph.trebesch@econ.lmu.de 